\section{(2) OPEN ACCESS}

\title{
Two-year outcomes of the MINlject drainage system for uncontrolled glaucoma from the STAR-I first-in- human trial
}

\author{
Philippe Denis, ${ }^{1}$ Christoph Hirneiß, ${ }^{2}$ Georges M Durr (10), ${ }^{3}$ Kasu Prasad Reddy, ${ }^{4}$

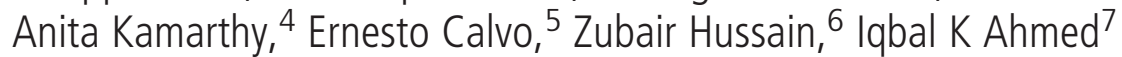

\begin{abstract}
- Supplemental material is published online only. To view please visit the journal online (http://dx.doi.org/10.1136/ bjophthalmol-2020-316888).

${ }^{1}$ Hôpital de la Croix-Rousse, Lyon, France

${ }^{2}$ Klinikum der

Universität München, Ludwig-Maximilians-

Universität, Munich, Germany ${ }^{3}$ Centre Hospitalier Universitaire de Montréal (CHUM), Montreal, Canada

${ }^{4}$ Maxivision Super Speciality Eye Hospital, Hyderabad, India ${ }^{5}$ Panama Eye Center (previously Clínica de Ojos OrillacCalvo), Panama City, Panama 6iSTAR Medical, Wavre, Belgium ${ }^{7}$ Ophthalmology and Vision Sciences, University of Toronto, Toronto, Canada
\end{abstract}

\section{Correspondence to}

Georges M Durr, Department of Ophthalmology, Centre Hospitalier Universitaire De Montréal (CHUM), 1051 Rue Sanguinet, Montreal, Quebec H2X 3E4, Canada; georgesdurr@gmail.com

Received 12 May 2020 Revised 7 September 2020 Accepted 16 September 2020

Check for updates

(C) Author(s) (or their employer(s)) 2020. Re-use permitted under CC BY-NC No commercial re-use. See rights and permissions. Published by BMJ.

To cite: Denis $P$, Hirneiß $C$, Durr GM, et al. Br J

Ophthalmol Epub ahead of print: [please include Day

Month Year]. doi:10.1136/

bjophthalmol-2020-

316888

\begin{abstract}
Background/Aims The current study evaluates the efficacy and safety of the stand-alone implantation of the MINIject (iSTAR Medical, Wavre, Belgium) supraciliary, microinvasive glaucoma drainage device in patients with medically uncontrolled open-angle glaucoma.

Methods This prospective, multicentre, first-in-human, single-arm interventional study evaluated stand-alone, ab interno implantation in 25 patients of a $5 \mathrm{~mm}$ long uveoscleral device made of STAR biocompatible material, which is a soft, microporous, flexible silicone. The primary outcome was the reduction of intraocular pressure (IOP) at 6 months compared with baseline, and follow-up continued until 2 years for 21 patients. Secondary outcomes included success defined as diurnal IOP of $\leq 21 \mathrm{mmHg}$ and $>5 \mathrm{mmHg}$ with an IOP reduction of $20 \%$ without (complete) or with/without (qualified) glaucoma medication.
\end{abstract}

Results Mean baseline IOP was $23.2 \pm 2.9 \mathrm{mmHg}$ on 2.0 \pm 1.1 glaucoma medication ingredients and decreased to $13.8 \pm 3.5 \mathrm{mmHg}(-40.7 \%$ reduction) on $1.0 \pm 1.3$ medications 2 years after implantation. Complete success was achieved in $47.6 \%$ of patients (10/21) and qualified success in $100 \%$ of patients $(21 / 21)$ at the 2 -year followup. All patients achieved a 20\% IOP reduction with $48 \%$ of patients medication-free. No serious ocular adverse events or additional glaucoma surgery were reported. Mean central endothelial cell density (ECD) mildly decreased from 2411 cells $/ \mathrm{mm}^{2}$ ( $\left.n=26\right)$ to 2341 cells/ $\mathrm{mm}^{2}(\mathrm{n}=21)$ at 24 months, which represents a $5 \%$ decrease for matched eyes. No patient had a $\geq 30 \%$ decrease in central ECD.

Conclusion This first-in-human study on the standalone implantation of the MINlject supraciliary drainage system shows promising IOP-lowering results and medication reduction over 24 months with few adverse events.

Trial registration number NCT03193736.

Glaucomatous vision loss remains one of the main reasons for blindness across the world. ${ }^{1}$ Traditionally, glaucoma is initially treated with topical drops, lasers and surgery as a last resort. All treatments are geared towards intraocular pressure (IOP) lowering. Given the significant morbidities associated with conventional glaucoma surgeries such as trabeculectomy and tube shunt procedures, minimally invasive glaucoma surgery (MIGS) was developed to help bridge the gap between topical/ laser therapy and traditional filters. The ideal MIGS procedure allows for moderate IOP lowering and a good safety profile, while causing minimal tissue disruption. $^{2}$ There are a variety of MIGS options available, which can be classified into different subtypes either targeting Schlemm's canal, supraciliary space or subconjunctival space.

The uveoscleral pathway accounts for $10-60 \%$ of aqueous outflow typically decreasing with age as extracellular fibrillar material accumulates in the ciliary muscle impeding aqueous drainage. ${ }^{3-5}$ Renewed interest in this space has led to the development of devices that help enhance outflow through the uveoscleral pathway. The CyPass Microstent (Alcon, Vernier-Geneva, Switzerland) and the iStent Supra (Glaukos Corporation, San Clemente, CA, USA) have both been designed to stent the suprachoroidal space using tubular implants delivered through an ab interno approach. ${ }^{6} 7$ The CyPass Microstent has recently been voluntarily withdrawn from the market due to concerns of endothelial cell loss as shown by the results of the COMPASS-XT trial, which was a 5-year extension of the initial pivotal COMPASS trial. ${ }^{6}$ Several studies have shown promising IOPlowering results with these suprachoroidal devices. $^{79}$

A novel implant has been developed targeting the suprachoroidal space: the MINIject ${ }^{\mathrm{TM}}$ glaucoma drainage device (iSTAR Medical SA, Wavre, Belgium). ${ }^{10}$ The device is inserted into the nasal quadrant of the eye through a $2 \mathrm{~mm}$ clear corneal incision. The MINIject implant is composed of a biocompatible porous silicone STAR ${ }^{\circledR}$ material. The unique flexible design conforms to the shape of the eye, and the micropores allow for enhanced aqueous outflow through the device. The STAR material has been shown to provide minimal tissue reaction, which is a promising feature of the implant given the risk of fibrosis. ${ }^{11}$ The MINIject is $5 \mathrm{~mm}$ in length with an oblong cross-sectional design measuring $1.1 \times 0.6 \mathrm{~mm}$ (figure 1). A green ring positioned at $0.5 \mathrm{~mm}$ from the tip of the device allows for accurate positioning in the anterior chamber. Preliminary 6-month results have been published previously. ${ }^{10}$ The purpose of the current study is to evaluate the efficacy and adverse events of the stand-alone implantation of the MINIject glaucoma drainage device with a 2-year follow-up, and final study results are reported in this manuscript. 


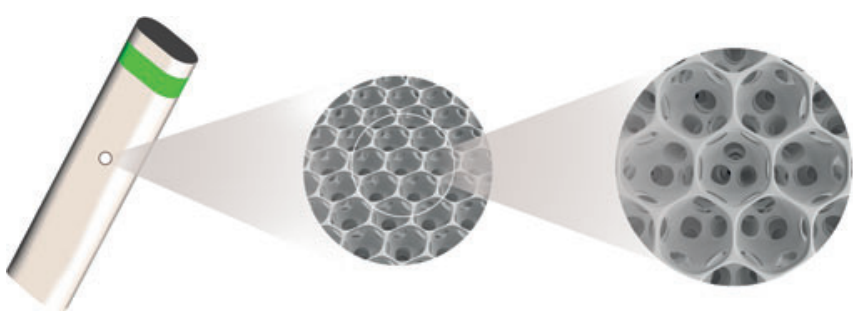

Figure 1 MINlject implant (iSTAR Medical, Wavre, Belgium) made of STAR material. Reprinted from Denis et al, ${ }_{1}^{10}$ copyright (2019), with permission from Elsevier and the American Academy of Ophthalmology.

\section{METHODS}

This paper reports 2-year outcomes of a multicentre, prospective, open-label, single-arm trial, in which patients were implanted with the MINIject glaucoma drainage device between June and October 2017 by three surgeons (PD, CH, IKA) in Panama and India. The study adhered to the tenets of the Declaration of Helsinki, and institutional review board approval was obtained from all concerned hospitals (Maxivision Super Speciality Eye Hospital, Hyderabad, India; Panama Eye Centre, formerly Clinica de Ojos Orillac-Calvo, Panama City, Panama). Written informed consent was provided by all participants of the study.

Patients included were at least 18 years old, had primary openangle glaucoma (POAG) with an angle grading of Schaffer 3 or 4 and IOP between $21 \mathrm{mmHg}$ and $35 \mathrm{mmHg}$ on one or more glaucoma medication classes. The main exclusion criteria were other types of glaucoma (angle closure, neovascular, uveitic), corneal or retinal disease, prior filtering glaucoma surgery and visual field (VF) defect within the central $10^{\circ}$. Methods were fully described in the previous preliminary paper. ${ }^{10}$

A comprehensive slit-lamp exam was performed to ascertain study eligibility with one eye per patient enrolled. In summary, the implantation consists of injecting the device through a $2 \mathrm{~mm}$ clear corneal incision in the nasal suprachoroidal space while using a direct view gonioprism and viscoelastic. The implant was advanced until the middle of the green ring coincided with the level of the scleral spur. No antimetabolites were used during or after implantation.

Postoperatively corticosteroid drops and antibiotic drops were used as per the investigator's discretion ( 25 of 26 eyes received topical antibiotics). Patients were seen following surgery at postoperative day 1 , weeks 1 and 2 and months $1,3,6,12,18$ and 24. All glaucoma medications were discontinued after the procedure. Glaucoma medications were re-introduced if IOP decreased $<20 \%$, or if IOP was at an unsafe level for the patient as per investigator discretion, or if glaucoma progression was noted on VF testing. IOP was measured at every visit with two readings obtained (a third was used if the two first readings differed by $>2 \mathrm{mmHg}$ ). Diurnal IOP (08:00, 12:00 and 16:00) was measured at baseline, 6, 12, 18 and 24 months. Preoperative baseline data including demographics (age, gender, ethnicity), as well as ocular characteristics and history (glaucoma medications; glaucoma diagnosis and severity; cup-to-disc ratio; best-corrected visual acuity (BCVA) using ETDRS chart at $4 \mathrm{~m}$; perimetry mean deviation (MD); history of laser trabeculoplasty, cataract surgery) were collected. VF testing using Humphrey Field Analyser (Carl Zeiss Meditec AG, Jena, Germany) was performed at baseline and months $1,3,6,12,18$ and 24 with progression qualified as a loss of $3 \mathrm{~dB}$ or more from baseline MD. Other testing included optic nerve examination and photography, ultrasound biomicroscopy (UBM) assessing implant position, and specular microscopy at multiple time points. Adverse events were recorded for each patient, specific ocular symptoms were questioned at each visit (blurred vision, glare, halos, dryness and foreign-body sensation) and patients were asked to complete seven questions from the Collaborative Initiative Glaucoma Treatment Study (CIGTS) quality of life patient questionnaire at baseline and at the 6-, 12-, 18- and 24-month follow-up visits (online supplemental appendix 1). ${ }^{12}$

The primary outcome of the study was reduction of IOP at 6 months. Secondary outcomes included success defined as diurnal IOP of $\leq 21 \mathrm{mmHg}$ and $>5 \mathrm{mmHg}$ with an IOP reduction of $20 \%$ without the use of glaucoma medication (complete success) or regardless of the use of glaucoma medication (qualified success). Other secondary outcomes included reduction of IOP at other follow-up time points, reduction in glaucoma medication and adverse events. Results were reported based upon the intention-to-treat population. These results were further confirmed in a separate analysis of the per-protocol population. Data analysis was performed using SAS version 9.3, concomitant medications were classified using the WHO Drug Dictionary (2017 V1) and adverse events coded using the Medical Dictionary for Regulatory Activities (MedDRA Version 20.0).

\section{RESULTS}

There were 43 patients screened for enrolment with 26 meeting all inclusion criteria and included in the safety population. One enrolled patient did not receive the implant due to anatomical concerns at the time of implantation in a myopic eye. Thus, 25 eyes were implanted with the device. Twenty-one patients completed the 24-month follow-up, two patients died (unrelated to the study implant), one was lost to follow-up and one withdrew from the study without providing a reason.

In the safety population, patients had a mean age \pm SD of 69.4 \pm 11.1 years with $50 \%(n=13)$ pseudophakic and $58 \%(n=15)$ were using two or more glaucoma medication ingredients. Glaucoma severity was assessed as mild or moderate in $88.4 \%$ $(n=23)$ with an MD of $-8.9 \pm 6.9 \mathrm{~dB}$. All patients had primary POAG with no history of laser trabeculoplasty. Baseline mean $\log$ MAR BCVA was $1.1 \pm 0.2$. Additional demographic variables can be found in the previously published report. ${ }^{10}$ Mean baseline IOP was $23.2 \pm 2.9 \mathrm{mmHg}$ using a mean of $2.0 \pm 1.1$ glaucoma medications. The primary endpoint at 6 months was met, with a signification reduction in IOP of $9.0 \mathrm{mmHg} .{ }^{10}$

\section{Results at 24 months}

The mean reduction in IOP 24 months after surgery was $40.7 \%$ compared with baseline, to a mean diurnal IOP of 13.8 $\pm 3.5 \mathrm{mmHg}$ at 24 months. At months 6,12 and 18, postoperative IOP decreased to $14.2 \pm 4.7 \mathrm{mmHg}, 16.0 \pm 4.8 \mathrm{mmHg}$ and 14.7 $\pm 3.5 \mathrm{mmHg}$, respectively. Glaucoma medications decreased to a mean of $1.0 \pm 1.3$ at 24 months, with $47.6 \%$ of patients $(10 / 21$ patients) medication-free. IOP and medication use throughout the follow-up period can be found in table 1 and figure 2. Qualified success was reached by $100 \%$ of patients $(21 / 21)$ and complete success by $47.6 \%$ of patients (10/21). If applying a lower IOP cutoff of $\leq 18 \mathrm{mmHg}$ in these definitions, qualified success would be $95.2 \%$ of patients (20/21) and complete success would be $42.9 \%$ (9/21) at the 24-month follow-up visit. Example UBM images can be found in figure 3. Furthermore, no additional glaucoma surgery was performed up to 24 months after MINIject implantation.

\section{Quality of life}

The results of the quality of life questionnaire show an absence or reduction in incidence for all symptoms at 24 months compared 
Table 1 Baseline and postoperative intraocular pressure (IOP) values and number of medications used in the intention-to-treat population

\begin{tabular}{|c|c|c|c|c|c|}
\hline & Baseline & 6 months & 12 months & 18 months & 24 months \\
\hline $\mathrm{n}$ & 25 & 24 & 24 & 22 & 21 \\
\hline IOP (mmHg), mean (SD) & $23.17(2.91)$ & $14.23(4.74)$ & $16.00(4.8)$ & $14.74(3.49)$ & $13.76(3.46)$ \\
\hline IOP reduction $(\mathrm{mmHg})$, mean $(\mathrm{SD})$ & NA & $8.95(3.69)$ & $7.17(4.63)$ & $8.51(3.79)$ & $9.55(3.84)$ \\
\hline IOP reduction $(\%)$, mean & NA & 39.07 & 30.83 & 36.24 & 40.65 \\
\hline Medication-free eyes, number (\%) & 0 & $21(87.5)$ & $19(79.1)$ & $14(63.6)$ & $10(47.6)$ \\
\hline Medications per eye, mean (SD) & $2.0(1.06)$ & $0.3(0.74)$ & $0.4(0.88)$ & $0.7(1.09)$ & $1.0(1.3)$ \\
\hline
\end{tabular}

with those reported at baseline, indicating an improvement in patient quality of life. In particular, there were significant reductions (75-100\%) in eye irritation/burning, eye pain, and skin sensitivity or irritation around the eye. There were also reductions (39-67\%) in excessive tearing, red eyes and the sensation of a foreign presence in the eye. Outcomes from the quality of life questionnaire can be found in table 2 .

\section{Adverse events}

A complete list of ocular adverse events in the study eye can be found in table 3 . The most frequent adverse events were anterior chamber reaction (8/26 patients, $30.8 \%)$, visual acuity reduced (8/ $26,30.8 \%)$, VF defect $(7 / 26,26.9 \%)$, IOP elevation $(5 / 26,19.2 \%)$ and lenticular opacities (5/26, 19.2\%). Visual acuity decreased nine times in eight patients with five events qualified as unrelated to the procedure and four possibly/probably related to the procedure (none related to the device). Two-thirds (6/9) of visual acuity reduction events resolved with cataract surgery, medications or spontaneously. Three patients had ongoing visual acuity reduction

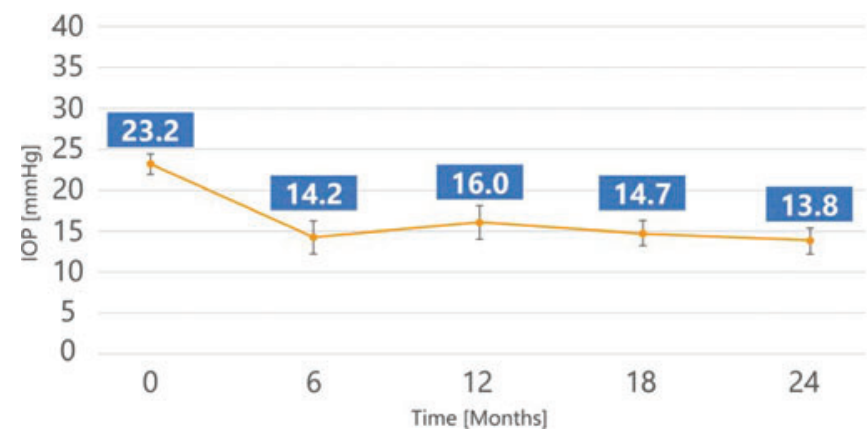

Figure 2 Mean intraocular pressure $(\mathrm{mmHg})$ with $95 \% \mathrm{Cl}$ error bars from baseline to 24-month follow-up in the intention-to-treat population. at the conclusion of the study of which two were related to cataracts (reduction of 20 letters and counting fingers) and one related to corneal oedema (counting fingers). The patient with corneal oedema had a preoperative endothelial cell density (ECD) count of 792 cells $/ \mathrm{mm}^{2}$ due to a complicated cataract surgery several years prior causing endothelial trauma. The investigator expected corneal decompensation to continue over time. Corneal oedema developed in the central area (away from the MINIject implantation site) 24 months post-surgery, and the investigator assessed oedema as being unrelated to the study device. Mean VF was unchanged between baseline (MD: $-8.9 \mathrm{~dB}$, pattern $\mathrm{SD}$ (PSD): 5.0) and 24-month follow-up (MD: $-8.7 \mathrm{~dB}$, PSD: 5.5 ). VF loss of $\geq 3 \mathrm{~dB}$ was reported in seven patients varying from mild to moderate with $4 / 7$ patients subsequently regaining the loss reported in their prior VF tests. Of these four patients, two had gains from cataract surgery and two patients were given medication or had spontaneous gains in VF. At the conclusion of the study, 3/7 patients had an ongoing VF loss: one patient with corneal oedema could not complete VF testing at 24 months and two patients had cataract progression with no surgery performed by study completion. Only one patient had IOP elevation $\geq 10 \mathrm{mmHg}$ from baseline (after the 1-month follow-up visit), which met the protocol definition of substantial IOP increase. All IOP elevations resolved. One patient developed mild, transient hypotony on postoperative day 2, which resolved within 7 days. There were no changes in mean refraction in the study eye. There were no suprachoroidal haemorrhages and no additional glaucoma surgeries were required.

Mean central ECD decreased from 2411 cells $/ \mathrm{mm}^{2}(\mathrm{n}=26)$ at baseline to 2341 cells $/ \mathrm{mm}^{2}(\mathrm{n}=21)$ at 24 months, representing a 5\% decrease for matched patients. At 24-month follow-up, there were no patients with a central ECD loss of $>=30 \%$ from baseline. BCVA and mean refraction remained the same throughout the study (mean logMAR BCVA change of $0.02 \pm 0.3$ and mean change in refraction of $-0.21 \pm 1.03 \mathrm{D})$. Two patient deaths

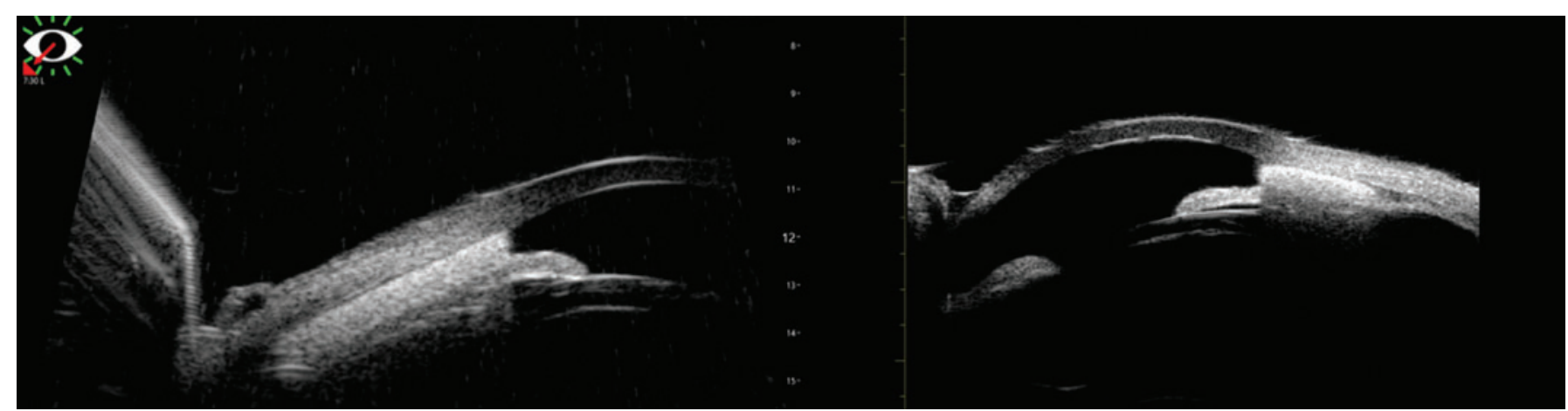

Figure 3 Example ultrasound biomicroscopy images at week 1 (left) and 24-month (right) follow-up. 
Table 2 Results of the quality of life patient questionnaire: whether the listed symptom was experienced in the last 7 days

\begin{tabular}{lll}
\hline Symptom & Baseline $(\mathrm{n}=\mathbf{2 6})$ & $\mathbf{2 4}$ months $(\mathrm{n}=\mathbf{2 1})$ \\
\hline Eye irritation/burning, $\mathrm{n}(\%)$ & $5(19)$ & $0(0)$ \\
\hline Eye pain, $\mathrm{n}(\%)$ & $5(19)$ & $1(5)$ \\
\hline Excessive tearing, $\mathrm{n}(\%)$ & $8(31)$ & $4(19)$ \\
\hline Droopy eyelid, $\mathrm{n}(\%)$ & $0(0)$ & $0(0)$ \\
\hline Red eye, $\mathrm{n}(\%)$ & $5(19)$ & $2(10)$ \\
\hline $\begin{array}{l}\text { Feeling like something is in the eye, } \mathrm{n}(\%) \\
\text { Skin sensitivity or irritation around the } \\
\text { eye, } \mathrm{n}(\%)\end{array}$ & $\mathbf{3 ( 1 5 )}$ & $1(5)$ \\
\hline
\end{tabular}

Table 3 Number of patients in the safety population experiencing adverse ocular events in the study eye from post-surgery until study completion

\begin{tabular}{ll}
\hline Adverse event & $\mathbf{n}(\%)$ \\
\hline Anterior chamber inflammation & $8(30.8)$ \\
\hline Visual acuity reduced & $8(30.8)$ \\
\hline Visual field defect & $7(26.9)$ \\
\hline Intraocular pressure increased* & $5(19.2)$ \\
\hline Lenticular opacities & $5(19.2)$ \\
\hline Hyphaema & $3(11.5)$ \\
\hline Conjunctival haemorrhage & $2(7.7)$ \\
\hline Eye pain & $2(7.7)$ \\
\hline Eye pruritus & $2(7.7)$ \\
\hline Cataract & $1(3.8)$ \\
\hline Cataract subcapsular & $1(3.8)$ \\
\hline Conjunctival oedema & $1(3.8)$ \\
\hline Corneal disorder & $1(3.8)$ \\
\hline Corneal oedema & $1(3.8)$ \\
\hline Cystoid macular oedema & $1(3.8)$ \\
\hline Detached Descemet's membrane & $1(3.8)$ \\
\hline Glare & $1(3.8)$ \\
\hline Hypotony of eye & $1(3.8)$ \\
\hline latrogenic corectopia & $1(3.8)$ \\
\hline Macular fibrosis & $1(3.8)$ \\
\hline Posterior capsular opacification & $1(3.8)$ \\
\hline Punctate keratitis & $1(3.8)$ \\
\hline Vitreous haemorrhage & $1(3.8)$ \\
\hline Corneal abrasion & $1(3.8)$ \\
\hline Conjunctival foreign body & $1(3.8)$ \\
\hline Enlyopaten & \\
\hline
\end{tabular}

* Only one patient had a mean intraocular pressure (IOP) increase, which fulfilled the protocol definition of substantial IOP increase ( $\geq 10 \mathrm{mmHg}$ higher than screening IOP after the

1-month postoperative visit).

$\mathrm{n}=$ number of patients.

during the follow-up period were deemed unrelated to the study (one died during sleep and one died as a result of pancreatic cancer). This was confirmed by the study's independent Safety Monitoring Committee. Overall, there were no untoward safety concerns related to the device during the 24-month study.

\section{DISCUSSION}

This prospective, first-in-human, multicentre, single-arm study examined the efficacy, success, adverse events and the safety profile of a novel supraciliary implant, MINIject. At 24 months, the patients in this study had a mean reduction in IOP of $40.7 \%$
(9.6 $\mathrm{mmHg}$ ), from medicated mean diurnal IOP of 23.2 $\pm 2.9 \mathrm{mmHg}$ at baseline to $13.8 \pm 3.5 \mathrm{mmHg}$ at 24 months A reduction of glaucoma medications was also shown from a mean of $2.0 \pm 1.1$ to $1.0 \pm 1.3$, with $47.6 \%$ of patients being medication free. Success, as defined by an IOP of $\leq 21 \mathrm{mmHg}$ and $>5 \mathrm{mmHg}$ with an IOP reduction of $20 \%$, was $47.6 \%$ for complete success (no glaucoma medications) and 100\% for qualified success (with and without glaucoma medications). Studies have shown that when IOP has been significantly reduced to levels $\leq 18 \mathrm{mmHg}$, glaucoma progression could potentially be stopped and further damage to the optic nerve prevented. ${ }^{13}{ }^{14}$ In addition, the CIGTS showed that IOP reductions of $35 \%$ and $48 \%$ in a 5 -year study were able to slow the progression of glaucoma. ${ }^{15}$ In this study, $95.2 \%$ of patients had IOP $\leq 18 \mathrm{mmHg}$ at 24 -month follow-up and there was a mean reduction in IOP of $40.7 \%$.

The IOP reduction and success rates in this study are considerable compared with other stand-alone MIGS devices targeting Schlemm's canal or the supraciliary space. As MINIject was implanted in a stand-alone procedure, it is more appropriate to compare these results with other stand-alone studies. Cataract surgery is a potential confounder. Supraciliary devices provide the best comparators given the similar implantation location. Stand-alone CyPass (Alcon) implantation $(n=65)$ was shown at 2 years in the DUETTE study to decrease IOP from a mean of $24.5 \pm 2.8 \mathrm{mmHg}$ to $16.8 \pm 3.9 \mathrm{mmHg}(-31 \%$ reduction) $(\mathrm{n}=32)$ with $56.3 \%$ having a $20 \%$ reduction of IOP from baseline and a mean medication reduction from $2.2 \pm 1.1$ at baseline to 1.5 \pm 1.2 at 2 years. ${ }^{16}$ At 2 years, 12 eyes $(18.5 \%)$ required additional glaucoma surgery. ${ }^{17}$ Success was defined as IOP between 6 and $21 \mathrm{mmHg}$ on no medications (complete success: 29.2\%) or on same or fewer medications (qualified success: $62.5 \%$ ). The 2-year results are limited by a significant loss to follow-up $(>50 \%)$. Another multicentre study examining real-world results for CyPass in the CyCLE trial $(n=224)$ found a baseline IOP of $22.6 \pm 6.7 \mathrm{mmHg}$ on a mean of $2.2 \pm 1.2$ medications decreasing to $16.7 \pm 3.8 \mathrm{mmHg}(-18 \%$ reduction) on $1.8 \pm 1.2$ medications at 2 years $(\mathrm{n}=120)$ with $58 \%$ achieving $20 \%$ decrease in IOP from baseline. At 3 years, 32\% required secondary glaucoma surgery. ${ }^{18}$

Trabecular bypass stents can also serve as comparators although they target the conventional outflow pathway. The recent COMPARE trial ${ }^{19}$ compared stand-alone 12-month outcomes of two iStent Trabecular Micro Bypass devices (Glaukos, San Clemente, CA, USA) with one Hydrus Microstent (Ivantis, Irvine, CA, USA). At baseline, mean medicated IOP was 19.0 \pm 3.9 (Hydrus, $\mathrm{n}=77$ ) and $19.1 \pm 3.6 \mathrm{mmHg}(2$-iStent, $\mathrm{n}=77)$ on a mean of $2.5 \pm 0.7$ (Hydrus) and $2.7 \pm 0.8$ (2-iStent) medications. At 12 months, mean IOP decreased to $17.3 \pm 3.7$ (Hydrus) and $18.1 \pm 3.7 \mathrm{mmHg}$ (2-iStent) on $1.0 \pm 0.3$ (Hydrus) and 1.7 \pm 0.3 (2-iStent) medications, and these results were sustained to 24 months. At 24 months, the unpublished data made available on the company website states that $63 \%$ of of patients in the Hydrus group compared to $40 \%$ of of patients in the 2 -iStent group obtained a $20 \%$ IOP reduction. Also, medication use decreased by a mean of 1.3 medications (52\%) with $38 \%$ of patients medication-free in the Hydrus group, compared to a reduction of 0.8 medications (29\%) with 19\% of patients medication-free in the 2-iStent group. No patients in the Hydrus group required reoperation as opposed to $9 \%$ in the 2-iStent group. ${ }^{20}$ There are many iStent studies that have shown varied amounts of IOP and medication reduction with standalone implantation. ${ }^{21-26}$

Another stand-alone study comparing Hydrus to selective laser trabeculoplasty up to 1 year found similar results with stand- 
alone Hydrus implantation $(n=31)$ with a reduction of IOP from $23.1 \pm 5.1 \mathrm{mmHg}$ on $2.3 \pm 0.8$ medications at baseline to 16.5 $\pm 2.6 \mathrm{mmHg}$ ( $26 \%$ reduction) on $0.9 \pm 1.0$ medications, although at only 12 -month follow-up, with $47 \%$ remaining medication free. $^{27}$

In comparison to these supraciliary and trabecular bypass studies, in our sample, mean baseline IOP was $23.2 \pm 2.9 \mathrm{mmHg}$ on $2.0 \pm 1.1$ glaucoma medications and decreased to 13.8 $\pm 3.5 \mathrm{mmHg}$ (40.7\% reduction) on $1.0 \pm 1.3$ glaucoma medications. All patients achieved at least 20\% IOP reduction with $48 \%$ of patients medication-free at the 2-year follow-up. In addition, no patients required further glaucoma surgery. Our study results show a trend towards considerably greater IOP reduction sustained up to a follow-up period of 24 months, with similar medication reduction, compared to the studies listed earlier.

Aside from stenting procedures, trabeculotomies have also shown similar IOP-lowering capabilities using devices such as the Trabectome (MicroSurgical Technology, Redmond, WA, USA) and the Kahook Dual Blade (KDB) (New World Medical, Rancho Cucamonga, CA, USA). A review article of the Trabectome literature has shown that the stand-alone procedure decreased IOP by a weighted mean difference of -9.8 $\pm 0.9 \mathrm{mmHg}$ with $-1.1 \pm 0.5$ less glaucoma medications at 2 years. ${ }^{28}$ The overall success rates using final IOP $\leq 21 \mathrm{mmHg}$ and $20 \%$ decrease in IOP from baseline were $61 \pm 17 \%$ at 1 year and $46 \pm 34 \%$ at 2 years. There was no indication of medication use to qualify success in these studies. Another stand-alone KDB study $(n=16)$ with only 12-month follow-up was shown to decrease IOP and medications from a baseline $20.4 \pm 1.3 \mathrm{mmHg}$ on $3.1 \pm 0.2$ medications to $14.1 \pm 0.9 \mathrm{mmHg}$ on $2.3 \pm 0.4$ medications. ${ }^{29}$ Complications such as hyphemas (up to $100 \%$ in Trabectome studies) and sustainability concerns are potential drawbacks to these procedures.

Finally, subconjunctival MIGS have also been studied as a standalone procedure. A prospective 2-year, multicentre series on the Xen gel stent (Allergan, Dublin, Ireland) has shown in stand-alone implantation $(n=106)$ a reduction of IOP from a baseline of 21.7 $\pm 3.8 \mathrm{mmHg}$ on $2.7 \pm 0.9$ medications to $15.4 \pm 5.2 \mathrm{mmHg}$ (28.2\% reduction) on $1.2 \pm 1.2$ medications. ${ }^{30}$ Of note, subconjunctival MIGS have several significant differences compared with other ab interno MIGS, as they completely bypass the conventional and uveoscleral pathways of aqueous humour drainage by creating a filtering bleb with mitomycin use and potential risks of infection and hypotony. They also require a more intensive postoperative follow-up and potential needling (41\%). ${ }^{30}$

Investigators from the CIGTS have suggested that quality-oflife perception may be even more important for patients than small differences in treatment results, even if those differences are statistically or clinically significant. The quality of life questionnaire completed by patients in this study showed an improvement or absence in all areas measured. ${ }^{12}$ In particular, there were marked reductions (50-100\%) in eye irritation/burning, eye pain and excessive tearing, which could be attributed to the reduction in medication burden with around half of the patients on no medications at final follow-up. Also, a reduction in foreignbody sensation $(75 \%)$, and skin sensitivity or irritation around the eye $(100 \%)$ were noted.

There were few adverse events during the study. The most common ones were transient inflammation and IOP elevations. These are quite common after MIGSs and resolved with conservative therapy or spontaneously. Cataract surgery is often anticipated when performing MIGS in a phakic patient, particularly since some patients had pre-existing cataracts at baseline, and considering the mean age of patients and the duration of the study. Indeed, $3 / 13$ phakic patients $(23 \%)$ in our study had cataract surgery in the study eye during the study period. Of these three eyes, two had pre-existing mild/ moderate cataracts at baseline. In comparison, Grisanti et al reported 19/75 phakic patients (25\%) had cataract surgery up to 2 years after CyPass implantation. ${ }^{18}$ The DUETTE study found $11 / 41$ phakic patients $(27 \%)$ had cataract surgery up to 24-month follow-up. ${ }^{17}$ Another potential concern is ECD loss, as seen with the CyPass device, which ultimately led to its withdrawal from the market. ${ }^{31}$ ECD results after MINIject implantation have shown minimal ECD loss at 2 years, which is encouraging. This could be due to MINIject's soft and conforming material, as well as its design which minimises the amount of material in the anterior chamber $(0.5 \mathrm{~mm})$. ECD results in this study will continue to be monitored up to 5 years. Being an ab interno, bleb-free, supraciliary procedure, there were no adverse events associated with blebs, needling or revision surgery, and no MMC-related complications due to the prohibition of MMC use per study protocol.

Despite the fact that a direct comparison is challenging, these data indicate that MINIject has shown a trend towards greater IOP and medication reduction compared to current MIGS options, with results sustained out to 24 months. These promising results were achieved alongside improved quality of life indicators, with no untoward safety concerns, and with minimal reduction in ECD levels.

Limitations of this study include its non-comparative design. Overall, it is difficult to compare all these different procedures by simply looking at IOP and medication reduction, due to many different factors that affect surgical success including age, disease severity, glaucoma type, surgical technique, preoperative IOP, preoperative medication use, etc. The small sample size and limited sites/surgeons decrease the generalisability of the results, which is common in first-in-human trials. There were no untoward safety concerns throughout the study. As is the case with many glaucoma trials, IOP is an imperfect measure of glaucoma control. VF testing was performed to assess progression during the trial, but there was a significant learning curve for patients, and testing was not systematically repeated in case of significant VF changes.

This first-in-human study on the MINIject drainage system shows significant IOP and medication reduction over 24 months with few adverse events. The risk of fibrosis and device failure is an ongoing concern with any glaucoma surgery. The proprietary STAR material has shown a minimal inflammatory response in rabbits ${ }^{11}$, supported clinically by the results in this study which show sustained IOP lowering up to 24 months. As with any MIGS device, these results need to be confirmed with a larger sample of patients and long-term studies.

Acknowledgements The study investigators would like to thank the independent members of the Safety Monitoring Committee: A.G. Jünemann MD (Rostock University, Rostock, Germany); R.D. Fechtner MD (SUNY Upstate Medical University, NY, USA); R A. Eiferman MD (University of Louisville, Louisville, USA); L.H. Silver PhD (Silver Pharma Consulting Inc., El Paso, Texas, USA). The authors acknowledge statistical analyses by Nicolas Dubois, MSc, and Koenraad D'Hollander, MD, MSc (IDDI, Louvain-la-Neuve, Belgium), clinical operations support by Ljubomir Manola, PhD (iSTAR Medical, Wavre, Belgium), and writing and editorial assistance provided by Katherin Awad, BSC, MBA.

Funding iSTAR Medical (Wavre, Belgium) has directly sponsored the STAR-I study. The sponsor participated in the design of the study, conducting the study and review of the manuscript. There is no grant number.

Competing interests PD: iSTAR Medical, Alcon, Allergan, Glaukos, Santen, Thea; CH: iSTAR Medical, Thea Pharma; GMD: Alcon, Allergan, Bausch \& Lomb, Glaukos, Labtician, Novartis, MST, Santen, Sight Sciences; KPR, AK, EC: no financial interests; ZH: Vice President of Regulatory and Clinical at iSTAR Medical; IKA: Aequus, Aerie Pharmaceuticals, Alcon, Allergan, ArcScan, Bausch \& Lomb, Beaver Visitec, Camras 
Vision, Carl Zeiss Meditec, CorNeat Vision, Ellex, ElutiMed, Equinox, Genentech, Glaukos, Gore, Iantech, InjectSense, Iridex, iSTAR Medical, Ivantis, Johnson \& Johnson Vision, KeLoTec, LayerBio, Leica Microsystems, MicroOptx, New World Medical, Omega Ophthalmics, PolyActiva, Sanoculis, Santen, Science Based Health, Sight Sciences, Stroma, TrueVision, Vizzari.

Provenance and peer review Not commissioned; externally peer reviewed.

Data availability statement Data are available upon reasonable request. Data may be obtained from a third party and are not publicly available.

Supplemental material This content has been supplied by the author(s). It has not been vetted by BMJ Publishing Group Limited (BMJ) and may not have been peerreviewed. Any opinions or recommendations discussed are solely those of the author(s) and are not endorsed by BMJ. BMJ disclaims all liability and responsibility arising from any reliance placed on the content. Where the content includes any translated material, BMJ does not warrant the accuracy and reliability of the translations (including but not limited to local regulations, clinical guidelines, terminology, drug names and drug dosages), and is not responsible for any error and/ or omissions arising from translation and adaptation or otherwise.

Open access This is an open access article distributed in accordance with the Creative Commons Attribution Non Commercial (CC BY-NC 4.0) license, which permits others to distribute, remix, adapt, build upon this work non-commercially, and license their derivative works on different terms, provided the original work is properly cited, appropriate credit is given, any changes made indicated, and the use is noncommercial. See: http://creativecommons.org/licenses/by-nc/4.0/.

\section{ORCID ID}

Georges M Durr http://orcid.org/0000-0002-0627-3662

\section{REFERENCES}

1 Tham YC, Li X, Wong TY, et al. Global prevalence of glaucoma and projections of glaucoma burden through 2040: a systematic review and meta-analysis. Ophthalmology 2014:121:2081-90.

2 Saheb H, Ahmed II. Micro-invasive glaucoma surgery: current perspectives and future directions. Curr Opin Ophthalmol 2012;23:96-104.

3 Becker B, Neufeld A. Pressure dependence of uveoscleral outflow. J Glaucoma 2002;11:545

4 Bill A. Some aspects of aqueous humour drainage. Eye 1993;7:14-19.

5 Alm A, Nilsson SF. Uveoscleral outflow: a review. Exp Eye Res 2009;88:760-8.

6 Vold S, Ahmed II, Craven ER, et al. Two-year COMPASS trial results: supraciliary microstenting with phacoemulsification in patients with open-angle glaucoma and cataracts. Ophthalmology 2016;123:2103-12.

7 Myers JS, Masood I, Hornbeak DM, et al. Prospective evaluation of two iStent((R)) trabecular stents, one istent supra((R)) suprachoroidal stent, and postoperative prostaglandin in refractory glaucoma: 4-year outcomes. Adv Ther 2018;35:395-407.

8 lanchulev T, Lane S, Masis M, et al. Corneal endothelial cell density and morphology after phacoemulsification in patients with primary open-angle glaucoma and cataracts: 2-year results of a randomized multicenter trial. Cornea 2019;38:325-31.

9 Vinod K. Suprachoroidal shunts. Curr Opin Ophthalmol 2018;29:155-61.

10 Denis $P$, Hirneiss $C$, Reddy K, et al. A first-in-human study of the efficacy and safety of MINlject in patients with medically uncontrolled open angle glaucoma (STAR-I). Ophthalmol Glaucoma 2019;2:290-7.

11 Grierson I, Minckler D, Rippy MK, et al. Pre-clinical experience of STAR material. European Society of Cataract and Refractive Surgery Annual Meeting. Vienna: Austria. 2018

12 Janz N, Wren P, Lichter P, et al. The collaborative initial glaucoma treatment study: interim quality of life findings after initial medical or surgical treatment of glaucoma. Ophthalmology 2001;108:1954-65.
13 The Advanced Glaucoma Intervention Study (AGIS). 7. The relationship between control of intraocular pressure and visual field deterioration. The AGIS Investigators. Am J Ophthalmol 2000;130:429-40.

14 Vogel R, Crick R, Newson R, et al. Association between intraocular pressure and loss of visual field in chronic simple glaucoma. Br J Ophthalmol 1990;74:3-6.

15 Lichter PR, Musch DC, Gillespie BW, et al. Interim clinical outcomes in the collaborative initial glaucoma treatment study comparing initial treatment randomized to medications or surgery. Ophthalmology 2001;108:1943-53.

16 Garcia-Feijoo J, Hoh H, Uzunov R, et al. Supraciliary microstent in refractory open-angle glaucoma: two-year outcomes from the DUETTE trial. J Ocul Pharmacol Ther 2018:34:538-42

17 García-Feijoo J, Rau M, Grisanti S, et al. Supraciliary micro-stent implantation for open-angle glaucoma failing topical therapy: 1-year results of a multicenter study. Am J Ophthalmol 2015;159:1075-81.

18 Grisanti S, Grisanti S, Garcia-Feijoo J, et al. Supraciliary microstent implantation for open-angle glaucoma: multicentre 3-year outcomes. BMJ Open Ophthalmol 2018;3: e000183.

19 Ahmed IIK, Fea A, Au L, et al. A prospective randomized trial comparing Hydrus and iStent microinvasive glaucoma surgery implants for standalone treatment of open-angle glaucoma: the COMPARE study. Ophthalmology 2020;127:52-61.

20 Ivantis announces 24-month results of landmark prospective, randomized comparative MIGS clinical trial. 2019. Available https://www.ivantisinc.com/newsroom/ivantisannounces-24-month-results/

21 Chang DF, Donnenfeld ED, Katz LJ, et al. Efficacy of two trabecular micro-bypass stents combined with topical travoprost in open-angle glaucoma not controlled on two preoperative medications: 3-year follow-up. Clin Ophthalmol 2017;11:523-8.

22 Lindstrom R, Lewis R, Hornbeak DM, et al. Outcomes following implantation of two second-generation trabecular micro-bypass stents in patients with open-angle glaucoma on one medication: 18-month follow-up. Adv Ther 2016:33:2082-90.

23 Berdahl J, Voskanyan L, Myers JS, et al. Implantation of two second-generation trabecular micro-bypass stents and topical travoprost in open-angle glaucoma not controlled on two preoperative medications: 18-month follow-up. Clin Exp Ophthalmol 2017:45:797-802

24 Fea AM, Belda Jl, Rekas M, et al. Prospective unmasked randomized evaluation of the iStent inject ((R)) versus two ocular hypotensive agents in patients with primary open-angle glaucoma. Clin Ophthalmol 2014;8:875-82.

25 Ahmed II, Katz LJ, Chang DF, et al. Prospective evaluation of microinvasive glaucoma surgery with trabecular microbypass stents and prostaglandin in open-angle glaucoma. J Cataract Refract Surg 2014;40:1295-300.

26 Vold SD, Voskanyan L, Tetz M, et al. Newly diagnosed primary open-angle glaucoma randomized to 2 trabecular bypass stents or prostaglandin: outcomes through 36 months. Ophthalmol Ther 2016:5:161-72.

27 Fea AM, Ahmed II, Lavia C, et al. Hydrus microstent compared to selective laser trabeculoplasty in primary open angle glaucoma: One year results. Clin Exp Ophthalmol 2017:45:120-7.

28 Kaplowitz K, Bussel II, Honkanen R, et al. Review and meta-analysis of ab-interno trabeculectomy outcomes. Br J Ophthalmol 2016;100:594-600.

29 Sieck E, Epstein R, Kennedy J, et al. Outcomes of Kahook Dual Blade goniotomy with and without phacoemulsification cataract extraction. Ophthalmol Glaucoma 2018;1:75-81.

30 Reitsamer H, Sng C, Vera V, et al. Two-year results of a multicenter study of the ab interno gelatin implant in medically uncontrolled primary open-angle glaucoma. Graefes Arch Clin Exp Ophthalmol 2019;257:983-96.

31 Alcon announces voluntary global market withdrawal of CyPass Micro-Stent for surgical glaucoma. 2018. Available https://www.alcon.com/media-release/alconannounces-voluntary-global-market-withdrawal-cypass-micro-stent-surgical 